\title{
INDICATIONS FOR DENTAL TREATMENT UNDER GENERAL ANESTHESIA
}

\author{
Radosveta Andreeva \\ Department of Pediatric Dental Medicine, Faculty of Dental Medicine, \\ Medical University of Varna
}

\begin{abstract}
Pain control is a part of behavior management. If the pain is not controlled, it will affect the quality of work the dental practitioner can achieve. Dental treatment performed under general anesthesia allows dental practitioners to benefit from improved treatment conditions and provide better treatment. In addition, it also allows dentists to treat patients who otherwise cannot be treated in private dental offices, including children with high anxiety and/or phobias, poorly cooperative and non-cooperative children, patients with developmental disorders, patients with muscular control problems, patients with increased vomiting reflex. Many parents choose to treat their children under general anesthesia to reduce stress and increase comfort, and some children need this kind of treatment due to lack of cooperation due to early age, lack of maturity or physical/mental disorders.
\end{abstract}

Keywords: general anesthesia, indications, behavioral management

The indications for dental treatment under general anesthesia are based on specific criteria that take into account the risks, benefits, effectiveness, expected results, and the use of other behavioral management techniques as an alternative $(1,2)$. These indications require a competent assessment by the dentist. The decision to use general anesthesia is complicated by the presence of a small but real risk of complications or even, very rarely, death associated with the use of general anesthesia. The knowledge that most of the operative care can be performed using local

\footnotetext{
Address for correspondence:

Radosveta Andreeva

Faculty of Dental Medicine

Medical University of Varna

84 Tzar Osvoboditel Blvd

9000 Varna

e-mail:doctor_ra@abv.bg
}

Received: July 27, 2018

Accepted: September 19, 2018 anesthesia or behavioral management, places dental medicine away from other pediatric surgical specialties where general anesthesia is a traditional practice $(3,4,5)$. When discussing the use of general anesthesia with parents or guardians, some general considerations should be taken as important:

$\diamond$ Cooperative ability of the child;

$\diamond$ The degree of anxiety and how the child responds to such procedures;

$\diamond$ It is necessary to predict the extent of the surgical trauma;

$\diamond$ The complexity of the operative procedure $(6,7)$. The criteria used to select patients for dental treatment under general anesthesia are:

1. Patients who are moderately to extremely noncooperative due to:

$\diamond$ lack of psychological/emotional maturity;

$\diamond$ mental or physical medical disorders.

2. Patients with an allergy to local anesthesia or in cases when such an anesthesia cannot be 
effective due to acute infection or anatomic variation.

3. Children who are not capable of communicating verbally due to psychosocial, medical or cultural reasons.

4. Clinical cases, which require complex restorative and/or surgical procedures.

5. In cases when the use of general anesthesia can protect the developing psyche and/or reduce the medical risk.

6. Patients who need immediate and comprehensive oral/dental care (for example, dental abscess, endangering airways or other anatomical structures).

7. Children with behavioral problems that cannot be managed by the behavioral modeling techniques.

8. Patients who have multiple complicated caries and should be treated invasively for several appointments in the ambulatory.

9. Persons with difficult access to dental care $(8,9)$.

In specialized literature, the most common cause for dental treatment under sedation or general anesthesia in childhood is the non-cooperative behavior of the child. This lack of cooperation may be a consequence of various causes (age, intellectual or physical disorders). There is a worldwide lack of consensus on the criteria for the indication of pediatric dental treatment under general anesthesia. Several countries have in the recent years adopted an amendment to their legislation in this direction. The guidelines developed by the American Academy of Pediatric Dentistry (AAPD) in this regard are not with legislative authority, but rather consultative. They say:

"General anesthesia may be indicated for a patient who is unable to cooperate due to the child's age, anxiety/fear, degree of psychological maturity or the presence of medical, physical/mental impairment or delayed development. The decision to administer general anesthesia should take into account: the alternative ways and behavioral management options; dental treatment (range/need); the effect on the quality of dental treatment; the emotional status of the patient and it's medical condition." $(6,10)$

\section{INDICATIONS}

1. Mandatory indications of dental practitioners competence:

$\diamond$ Targeting a child for dental/surgical treatment under a sedation/general anesthesia is available only by a dental practitioner with a specialty in Pediatric Dentistry and after all other options have been exhausted. A single unsuccessful visit of a child to the dental office is not enough to target a child for sedation/general anesthesia.

$\diamond$ When a pediatric dentist (specialist) finds that, the child is non-cooperative during the preliminary examinations and an adequate treatment cannot be achieved with local anesthesia. This requires at least 3-4 visits to the pediatric dentist.

$\diamond$ For children with special needs, this is the only way to ensure quality dental treatment. The assessment and guidance could be done only by a dental practitioner with a specialty in Pediatric Dentistry $(11,12)$.

2. Mandatory indications of anesthetist's competence:

$\diamond$ Anesthesia standard equipment - for monitoring, oxygen sources, equipment for CPR-defibrilator, etc., complying with the requirements of the anesthesia and resuscitation standard $(10,13,14)$;

$\diamond$ Contract with a hospital with the appropriate childcare unit - Child Intensive Care Unit to take up any complications.

3. Recommended indications (circumstances and conditions suitable for general anesthesia):

a) According to the individual characteristics of the child patient:

$\diamond$ Non-cooperative children - due to lack of psychological or emotional maturity and/or mental, physical or medical disorders;

$\diamond$ Children with previous failed treatment with local anesthesia;

$\diamond$ Children/patients for whom local anesthesia is ineffective due to acute infection (inflammatory processes or traumas), anatomical characteristics;

$\diamond$ Children who could be protected from psychological trauma and/or have reduced medical 
risk in conditions that are potentially stimulated by stress by use of general anesthesia;

$\diamond$ Established allergy to local anesthetics.

b) Children with specific health needs. These are children who have or are at increased risk for disability. Children with special needs due to disability or chronic health problems who are eligible for treatment under general anesthesia are:

$\diamond$ Physical disorders - cerebral paralysis - severe form, muscular dystrophy, spina bifida;

$\diamond$ With severe intellectual disabilities, who need 24-hour care. These are patients with genetic disorders (Down syndrome), perinatal stroke, brain trauma. Many of these patients have other concomitant health problems - additional physical damage, epilepsy, neuropsychiatric problems, congenital heart defects or syndromes.

$\diamond$ With sensory impairments - when accompanying intellectual and physical disabilities in syndromes;

$\diamond$ Neuropsychiatric disorders - disorders from the autism spectrum and attention deficit hyperactivity disorder;

$\diamond$ Increased reflex for nausea and vomiting;

$\diamond$ Fear and behavioral problems;

$\diamond$ Focus with dental origin;

$\diamond$ Dentophobia (proven by a qualified specialist) $(6,13)$;

$\diamond$ Other - other medical conditions with oral manifestations which need to be treated in a hospital setting. The operating room is the best place to provide such care.

Severe pulpitis and acute infection are the most common diseases treated under general anesthesia $(15,3,7)$.

4. According to the range of dental treatment:

a) In case of a comprehensive pathology:

$\diamond$ Symptomatic teeth in more than one quadrant (multiple caries);

$\diamond$ Early childhood caries.

b) According to the severity of the pathology in severe pathology:

$\diamond$ Pulpitis and periodontitis requiring immediate relief due to severe pain;

$\diamond$ Acute swelling of soft tissues requiring removal of causal tooth/teeth; $\diamond$ Surgical drainage of abscess or phlegmones;

$\diamond$ Traumatic or complex extractions, e.g. in the case of ankylosed primary molars or primary molars in infraposition;

$\diamond$ Extraction of fractured permanent molars;

$\diamond$ Taking a biopsy of hard or soft tissues;

$\diamond$ Treatment or sewing of orofacial wounds;

$\diamond$ Postoperative bleeding requiring revision and suture;

$\diamond$ Single or multiple extractions of a child unsuitable for sedation;

$\diamond$ Teeth requiring surgical extraction or disclosure;

$\diamond$ Diagnostics including making X-rays for children with special needs where there is clinical evidence of need for dental treatment under general anesthesia $(14,16,17)$.

5. Contraindications for dental treatment under sedation/general anesthesia:

$\diamond$ Dental treatment and/or extractions with mild and moderate sedation of children up to 6 years of age;

$\diamond$ Healthy, cooperative patients with minimal needs (minimum range) of dental treatment;

$\diamond$ Carious, asymptomatic teeth without clinical or radiographic signs of inflammation;

$\diamond$ Extraction of healthy premolars by orthodontic indications in healthy children;

$\diamond$ Preference of the patient, parent, guardian, caregiver, except when other techniques have already been used;

$\diamond$ Predisposing medical conditions that would make general anesthesia undesirable (e.g., malignant hyperthermia, unstable cardiac status, poorly controlled cystic fibrosis) (18).

\section{REFERENCES}

1. Atan S, Ashley P, Gilthorpe MS, Scheer B, Mason C, Roberts G. Morbidity following dental treatment of children under intubation general anaesthesia in a day-stay unit. Int J Paediatr Dent. 2004; 14(1):9-16.

2. Cote CJ, Wilson S. American Academy of Pediatric Dentistry, American Academy of Pediatrics. Guidelines for monitoring and management of pediatric patients before, during, and after sedation 
for diagnostic and therapeutic procedures: Update 2016. Pediatr Dent. 2016;38(4):E19-E39.

3. American Society of Anesthesiologists. ASA physical status classification system. Available at: "http:/ www.asahq.org/ Accessed June 24, 2012.

4. Baakdah R, Frasi N, Boker A, Al Mushayt A. The use of general anesthesia in pediatric dental careof children at multi-dental centers in Saudi Arabia. J Clin Pediatr Dent. 2008; 33(2):147-53.

5. Chicka MC, Dembo JB, Mathu-Muju KR, Nash DA, Bush HM. Adverse events during pediatric dental anesthesia and sedation: A review of closed malpractice insurance claims. Pediatr Dent. 2012:34(3):231-8.

6. American Academy of Pediatric Dentistry. Policy on early childhood caries (ECC): classifications, consequences, and preventive strategies. Pediatr Dent. 2011; 35( 6):13 - 4.

7. AAPD. Guideline on behavior guidance for the pediatric dental patient. Pediatr Dent. 2011;33 (special issue):161-73.

8. Anand P, Wilson R, Sheehy EC. Intraligamental analgesia for post-operative pain control in children having dental extractions under general anaesthesia. Eur J Paediatr Dent. 2005; 6(1): 10-5.

9. DiMaggio C, Sun L, Li G. Early childhood exposure to anesthesia and risk of developmental and behavioral disorders in a sibling birth cohort. Anesth Analg. 2011;113(5): 1143-51.

10. AAPD. Policy on the use of deep sedation and general anesthesia in the pediatric dental office. Pediatr Dent. 2010; 30:64-5.

11. Bal N, Saricaoglu F, Uzun S, Dal D, Celebi N, Celiker $\mathrm{V}$, et al. Perioperative anxiety and postop- erative behavioural disturbances in children: comparison between induction techniques. Eur J Anaesthesiol. 2006; 23(6): 470-5. doi: 10.1017/ S0265021506000408.

12. Lin YT, Lin YTJ. Survey of comprehensive restorative treatment for children under general anesthesia. J Dent Sci. 2015;10(3):296-9. doi: 10.1016/j. jds.2014.09.002.

13. Blount RL, Loiselle KA. Behavioural assessment of pediatric pain. Pain Res Manag. 2009;14(1):47-52.

14. Kime SL, Wilson KE, Girdler NM. Evaluation of objective and subjective methods for assessing dental anxiety: a pilot study J Disability Oral Health. 2010; 11(2):69-76.15. AAPD. Oral health policy on hospitalization and operating room access for dental care of infants, children, adolescents, and persons with special health care needs. Pediatr Dent. 2010; 30(7 Suppl):68-9.

15. Knapp R, Gilchrist F, Rodd HD, Marshman Z. Change in children's oral health-related quality of life following dental treatment under general anaesthesia for the management of dental caries: a systematic review Int J Paediatr Dent. 2017;27(4):302-12. doi: 10.1111/ipd.12259.

16. Tjia I, Rampersad S, Varughese A, Heitmiller E, Tyler DC, Lee AC, et al. Wake Up Safe and root cause analysis: quality improvement in pediatric anesthesia.Anesth Analg. 2014;119(1):122-36. doi: 10.1213/ ANE.0000000000000266.

17. Ivanov K, Platikanov V. Sepsis - modern trends and methods of treatment. Collection of reports. Under order. by D. Damyanov. Pleven. 2010;38-44 (in Bulgarian). 